Revista Calidad en la Educación Superior

Programa de Autoevaluación Académica

Universidad Estatal a Distancia

ISSN 1659-4703

Costa Rica

revistacalidad@uned.ac.cr

EVALUACIÓN DE ASIGNATURAS DESDE LA PERSPECTIVA DE LOS ESTUDIANTES EN PROGRAMAS DE EDUCACIÓN SUPERIOR A DISTANCIA

COURSES'S EVALUATION IN HIGHER EDUCATION PROGRAMS FROM THE STUDENTS PERSPECTIVE

Jensy Campos Céspedes Licenciada en trabajo Social Máster en Evaluación de Programas y Proyectos

Estudiante de Doctorado en Tecnología Instruccional y Educación a Distancia de la Nova Southeastern University Universidad Estatal a Distancia San José, Costa Rica

Alejandro Sánchez Araya Licenciado en Educación Cívica Estudiante de la Maestría en Educación a Distancia Universidad Estatal a Distancia San José, Costa Rica

Volumen 1, Número 1 Mayo 2010

pp. $1-12$

Recibido: abril, 2010

Aprobado: mayo, 2010 


\section{EVALUACIÓN DE ASIGNATURAS DESDE LA PERSPECTIVA DE LOS ESTUDIANTES EN PROGRAMAS DE EDUCACIÓN SUPERIOR A DISTANCIA}

\section{Resumen}

El propósito de este artículo es compartir los resultados de una experiencia evaluativa realizada en una asignatura de investigación de la Cátedra de Investigación Educativa de la Universidad Estatal a Distancia (UNED) de Costa Rica. En el estudio participaron 126 estudiantes de los diferentes centros universitarios de la universidad y se basó en la aplicación de un cuestionario autoadministrado mediante el cual el estudiantado valoró la calidad de la asignatura desde los diferentes aspectos que lo componen. Los resultados se analizaron mediante estadísticas descriptivas utilizando el software spss. La evaluación concluyó que la valoración general que los estudiantes hacen de la asignatura es satisfactoria. No obstante, se detectan aspecto relacionados especialmente con los materiales que indican la necesidad realizar modificaciones de la asignatura para lograr un mejoramiento en términos de fortalecer los procesos de aprendizaje de los estudiantes.

\section{Palabras clave}

$\bullet$ Evaluación •Calidad •Educación a distancia •Opinión de estudiantes

\section{Summary}

The purpose of this article is to share the results of an evaluative experience conducted in a subject of investigation by the Educational Research Chair of the Universidad Estatal a Distancia (UNED) of Costa Rica. The study involved 126 students from different colleges of the university and was based on the application of a selfadministered questionnaire in which the students assessed the quality of the course from different aspects that comprise it. The results were analyzed by descriptive statistics using SPSS. The evaluation concluded that the overall assessment made by students of the subject is satisfactory. However, looks are detected especially related materials indicating the need for modifications of the subject to achieve an improvement in terms of strengthening the processes of student learning.

\section{Keywords}

Assessment • Quality • Distance Education • Student's Opinion 


\section{INTRODUCCIÓN}

Parecería ser que hablar de evaluación de cursos es un acto absurdo cuando los modelos curriculares y de diseño instruccional ubican la evaluación de los programas educativos como parte del diseño mismo del proceso educativo o propuesta curricular (Díaz, 2003; SINAES, 2009). No obstante, en la realidad, la evaluación de la calidad está ausente en muchas de las asignaturas o cursos que cada ciclo académico se ofertan en las instituciones de educación superior.

En la Universidad Estatal a Distancia de Costa Rica (UNED) la evaluación de asignaturas o cursos es "un proceso continuo, sistemático y permanente que permite el mejoramiento de los procesos académico...es responsabilidad del (la) profesor(a) Encargado de Cátedra, en coordinación con la persona Encargada de Programa" (UNED, 2009, p.14). Sin embargo, no son todas las cátedras las que cuentan con evaluación periódica de las asignaturas, pese a que "la cultura de la autoevaluación y acreditación de carreras se ha incorporado, aunque no con la rapidez esperada, en forma progresiva al medio universitario costarricense, contribuyendo a mejorar de manera creciente la calidad de los procesos educativos, en especial durante la última década" (Azze, 2007, p.3)

De acuerdo con González (2005) "la calidad de una carrera o programa de formación universitaria a distancia viene dada por la medida en que:

- sus objetivos y programa de estudios mantienen pertinencia con la misión de la universidad, adecuación a las necesidades sociales y coherencia con las exigencias de la profesión o área disciplinar.

- la organización y desarrollo de los procesos de enseñanza y aprendizaje, de investigación y de servicio a la comunidad son eficientes y eficaces en sus resultados.

- El programa muestra una capacidad y voluntad de mejorar permanente en sus procesos de autorregulación, para promover la calidad de la enseñanza y el aprendizaje." (p.3)

Aunque la anterior delimitación de la calidad no hace referencia explícita a los cursos o asignaturas, es claro que es precisamente en las asignaturas donde se concretan, en gran medida, la congruencia entre el plan de estudios y la misión institucional y, la organización y desarrollo de los procesos de enseñanza - aprendizaje. De ahí que los procesos de autoevaluación, acreditación y mejoramiento de la calidad en la institución suponen la incorporación de estrategias de mejora continua de las carreras y las asignaturas que las integran. De igual modo los procesos de evaluación de la calidad académica se deben realizar en diferentes niveles de especificidad, siendo la evaluación de los cursos uno de los niveles más específicos, pero de suma importancia para la calidad de la oferta educativa. 
Fig. 1

Dimensiones de la evaluación de la calidad académica

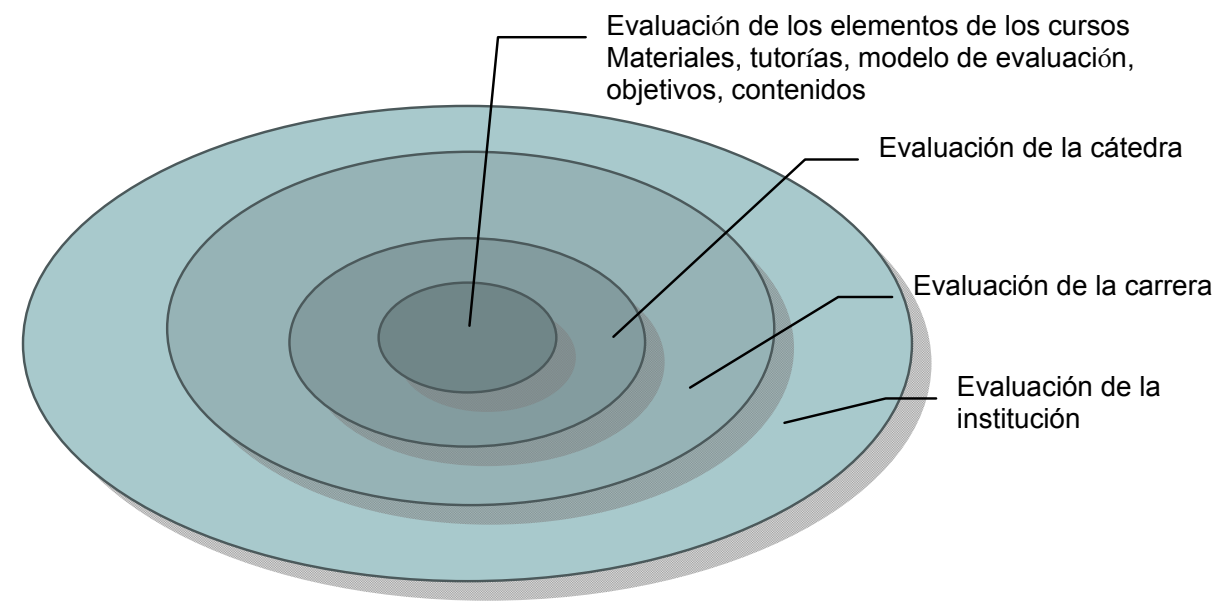

Fuente: elaboración propia, 2010.

Hablar de evaluación de asignaturas o cursos, en primera instancia, se debe hacer la siguiente reflexión: ¿cuál es el sentido de un curso en el plan de estudios de una carrera? La presencia de un curso en la malla curricular de una carrera debe tener una razón de ser, por ello los criterios para su diseño deben basarse en las áreas disciplinarias que componen el plan y en los elementos teóricos epistemológicos y ontológicos sobre los que se basa el plan de estudios. El criterio de pertinencia de la asignatura en función del perfil profesional y su adecuada ubicación en el plan de estudio, no son garantía de calidad suficiente del curso. Sino que las características de los estudiantes, la sincronía con el modelo pedagógico de la institución son, por ejemplo, otros criterios que deben tomarse en cuenta. Por ello es de suma importancia que las cátedras asuman la evaluación de cursos como práctica habitual para monitorear la suficiencia, consistencia, coherencia interna, actualidad y responder, al menos, a las interrogantes ¿En qué medida este curso es congruente con la carrera (s) de la cual(es) forma parte en su plan de estudios?, ¿En qué medida el curso es pertinente para las 
necesidades de la población estudiantil a la cual atiende? Y ¿En qué medida el curso es pertinente para el logro de los rasgos del perfil profesional de la carrera(s) a la cual sirve?

El desarrollo de los cursos en un plan de estudios de una carrera universitaria tiene la obligatoriedad de ajustarse a las necesidades y requerimientos de calidad con el fin de contribuir a la formación que la carrera propone brinda y la evaluación permite validar las propuestas instruccionales en cuanto a materiales, estrategias didácticas y pedagógicas brindadas; asimismo, permite identificar aspectos débiles en el diseño y ejecución del curso y delinear acciones de mejora para el desarrollo de la calidad en los cursos. Asimismo, la investigación y evaluación del quehacer permite a los formadores mejorar su práctica pedagógica Stenhouse (2003).

La evaluación del curso que se presenta en este documento se desprende de una propuesta de evaluación curricular planteada por Berrocal y Campos (2008) la que a su vez surge como parte de una experiencia de autoevaluación de un programa académico y atiende los requerimientos de información propios de los procesos de autoevaluación con fines de acreditación ante el Sistema Nacional de Acreditación de la Educación Superior (SINAES) y rescata los elementos cruciales establecidos por González (2006) referidos a "la planificación, la programación, los recursos, el desarrollo, y los resultados" (p.19).

Desde el punto de vista de la participación, dentro del proceso evaluativo, el modelo privilegia la participación de los actores educativos ya que reconoce la riqueza de los aportes de cada uno de ellos en la valoración y juzgamiento de la propuesta académica. En este sentido, la evaluación debe contar con la participación de al menos cuatro actores: el primero es el personal académico encargado de la coordinación de la carrera o programa, interesa que el énfasis de la valoración se centre en la pertinencia externa de un curso con respecto al plan de estudios y la coherencia interna entre los diferentes elementos que lo integran y la actualidad del mismo. El segundo actor evaluador está constituido por los docentes que participan en el desarrollo del curso. En tercer lugar se encuentran los estudiantes, quienes participan del desarrollo del curso y quienes constituyen el centro del proceso educativo (UNED, 2002) y en cuarto lugar se ubican el personal encargado o coordinador de la cátedra.

Fig. 2

Actores educativos que deben participar en la evaluación de cursos o asignaturas

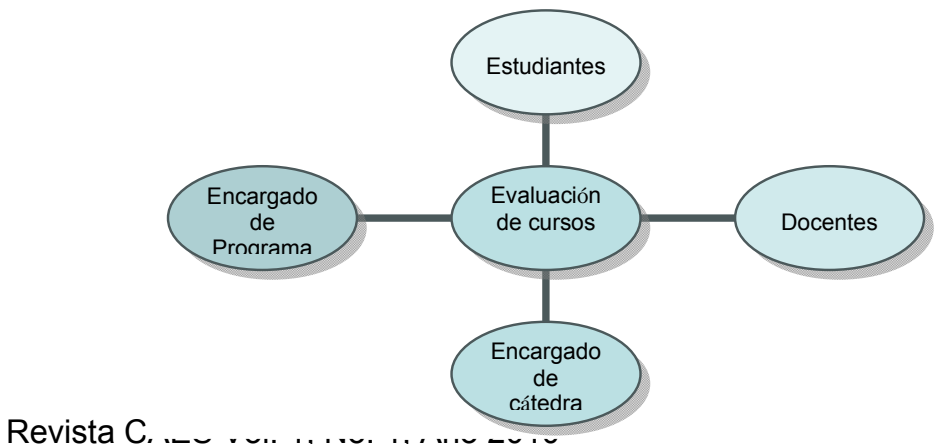

Revista C 
Fuente: Elaboración propia, 2010.

Dado que la opinión de los estudiantes respecto a aquellos elemento que pueden ayudar a determinar la calidad de un curso es fundamental y permite orientar las acciones de mejoramiento de la asignatura (Beverley, Tucker, Gupta \& Yeo, 2008) de allí que se destine este documento a recuperar los resultados de la evaluación realizada por el estudiantado.

De acuerdo con los planteamientos de González (2006) y de Berrocal \& Campos (2008) se derivan cinco elementos clave para la determinación de la calidad de los cursos que se brinda bajo la modalidad a distancia con tutoría presencial. A continuación se describe cada uno de ellos:

Los objetivos y los contenidos: Los objetivos o propósito del curso deben estar en función de las competencias, rasgos del perfil o habilidades, destrezas y conocimientos que se requieran desarrollar en el estudiante. Según González (2006) las asignaturas deben valorarse "tanto desde el punto de vista de los contenidos, como de los objetivos de aprendizaje y de las propuestas didácticas para su asimilación por el estudiante" (p.21).

Materiales didácticos. Los materiales se conciben como "un recursos que es cualitativamente medular" (González, 2006, p.21) y en educación a distancia el material se constituye en un elemento esencial para asegurar la calidad de los procesos de aprendizaje (Heinich, Molenda, Russell, \& Smaldino, 1999).

Evaluación de los aprendizajes. Los modelos de evaluación de aprendizaje tienen una fuerte incidencia en los procesos de aprendizaje de los estudiantes. Interesa especialmente valorar su congruencia dentro del contexto del curso, su relación con los resultados de aprendizaje que se planea alcanzar. Asimismo resulta importante conocer la contribución que permite la evaluación de los aprendizajes en función de promover en los estudiantes los aprendizajes cognitivos, el desarrollo de destrezas y la formación de actitudes positivas, así como el interés por el aprendizaje continuo y la construcción de un pensamiento crítico, creativo y autónomo (SINAES, 2009, p.61). 
Tutorías y asesorías académicas. Se incluye este elemento debido a que la modalidad a distancia la labor docente y la facilitación del aprendizaje es fundamental (Simonson, Smaldino, Albrigh \& Zvacek, 2006) y en la UNED la docencia "se constituye en lo expresión del desempeño del docente, en las asignaturas...el desempeño del docente en las tutorías y el acompañamiento que brinda al estudiante en su proceso de aprendizaje es vital...el docente se constituye en facilitador de los aprendizajes y la relación entre estudiante-docentes son diversas pero el propósito es dar seguimiento y atención durante el proceso de aprender, y no el proveer información, exponerla o demostrarla" (UNED, 2002, p.15).

De tal manera que la evaluación de cursos por parte del estudiantado deberá contemplar como mínimo los aspectos que se sintetizan en la siguiente figura.

Fig. 3

Aspectos que deben ser evaluados por los estudiantes en los proceso de evaluación de asignaturas

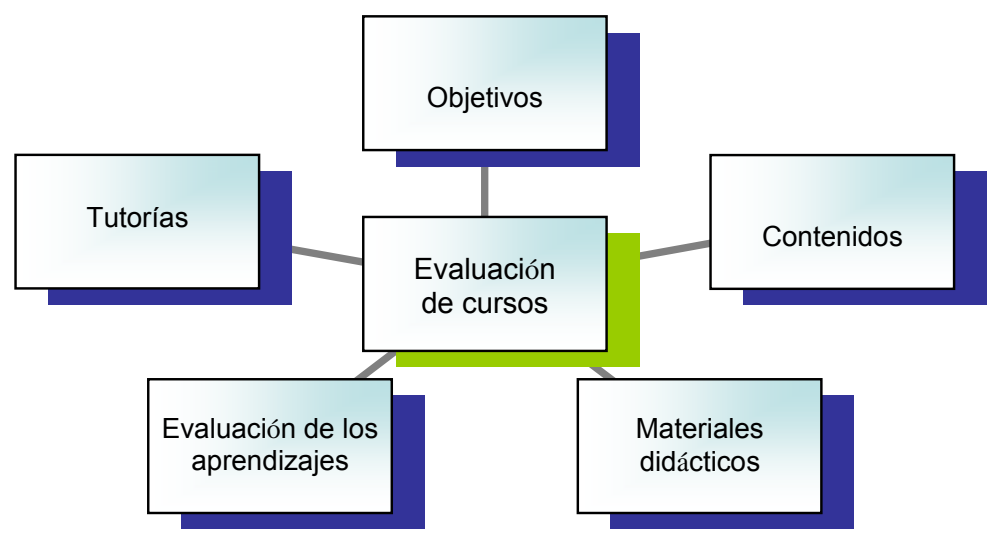

Fuente: Elaboración propia, 2010.

\section{METODOLOGÍA}

Para el desarrollo del estudio se utilizó como base el instrumento ofrecido por el Programa de Autoevaluación Académica (PAA), para evaluar cursos bajo la modalidad a distancia con tutorías presenciales.

La validación del instrumento. El instrumento fue sometido a validación por criterio de experto ante académicos con experiencia en educación a distancia, posteriormente, en el segundo y tercer cuatrimestre del 2008, se aplicó a 1287 estudiantes de diferentes cursos de la Escuela de Ciencias de la Educación (ECE). Se le aplicaron pruebas estadísticas para 
medir validez interna. El instrumento presentó un Alpha de Cronbach arrojó un 0. 896 por lo que se consideró que es bueno para medir los aspectos que se pretenden medir (George \& Mallery, 2008).

Descripción del instrumento. El instrumento fue diseñado para ser autoadministrado por los estudiantes. Se compone por una escala Likert de 34 reactivos que se encuentran agrupados dentro de las cinco categorías clave a evaluar, a saber; los objetivos y los contenidos, los materiales didácticos, la evaluación de los aprendizajes y las tutorías o asesorías académicas. Además, el instrumento incluye un ítem que consulta por la valoración general de la asignatura o curso.

Para el primer semestre del 2009, se aplicó el instrumento a un total de 126 estudiantes (distribuidos en diferentes centros universitarios de la institución universitaria) de uno de los cursos de la cátedra de investigación educativa. La administración del instrumento estuvo a cargo del personal de apoyo administrativo de los centros universitarios quienes, previa coordinación con la Dirección de Centros, se encargaron de la administración y devolución de los instrumentos debidamente completados a la cátedra.

Para procesar los datos, en primera instancia, se creó una base de datos en Excel y, posteriormente, se volcó la base de datos en el software SPSS versión 17.0 para la realización de procesamiento de datos que incluyó el tratamiento y cálculo de estadísticas descriptivas, cruce de variables y algunas pruebas de correlación entre variables que, de cuerdo con el criterio de experto de los investigadores, podrían estar asociadas.

Administración del instrumento. Para la aplicación del instrumento, se contó con la colaboración del personal de los diferentes centros universitarios. Se coordinó con la Dirección de Centros, se embalaron y se enviaron los instrumentos a los centros universitarios donde se aplicaron a los estudiantes durante la décima tutoría presencial del curso. La tasa de respuesta de los estudiantes asistentes a las tutoras fue de un $100 \%$ para cada uno de los reactivos que contiene el instrumento.

\section{ANÁLISIS Y DISCUSIÓN}

Los datos analizados corresponden a los instrumentos aplicados a 126 estudiantes del curso taller de investigación en diferentes centros universitarios del país.

La distribución de los participantes por centro universitario fue la siguiente: Liberia $11.9 \%$, Turrialba $20.6 \%$, Cartago $12.2 \%$, San José $7.1 \%$, Palmares $11.9 \%$, Alajuela $14.3 \%$, Heredia 19.8\%. La aplicación del instrumento se realizó a una muestra de 126 estudiantes de una población total de 281 de estudiantes matriculados en el curso. Asimismo, se dio el caso de un centro universitario que envió tardíamente los instrumentos; pese a ello la 
cantidad de participantes de este estudio es altamente representativo del total de la población matriculada en el curso evaluado y se abordan el $100 \%$ de centros universitarios en los que se desarrolla el curso.

Con respecto a las respuestas y calificaciones asignadas por los participantes en cada uno de los aspectos del curso, sometidos a valoración por parte de los estudiantes, se observó que el factor mejor calificado fue el de Tutorías y Asesorías Académicas, representados en los ítemes del 16 al 24 del instrumento. Para este factor, en la mayoría de los ítemes, la calificación de los participantes se ubicó en excelente. Entre el $78 \%$ y el 98\% de los participantes asignaron la mayor puntuación a los aspectos indicados en los ítemes del instrumento que se destinan a valorar la calidad de las tutorías.

\section{Gráfica 1.}

\section{Percepción de los estudiantes con respecto a las tutorías que recibe en el curso. Evaluación de curso, 2009.}

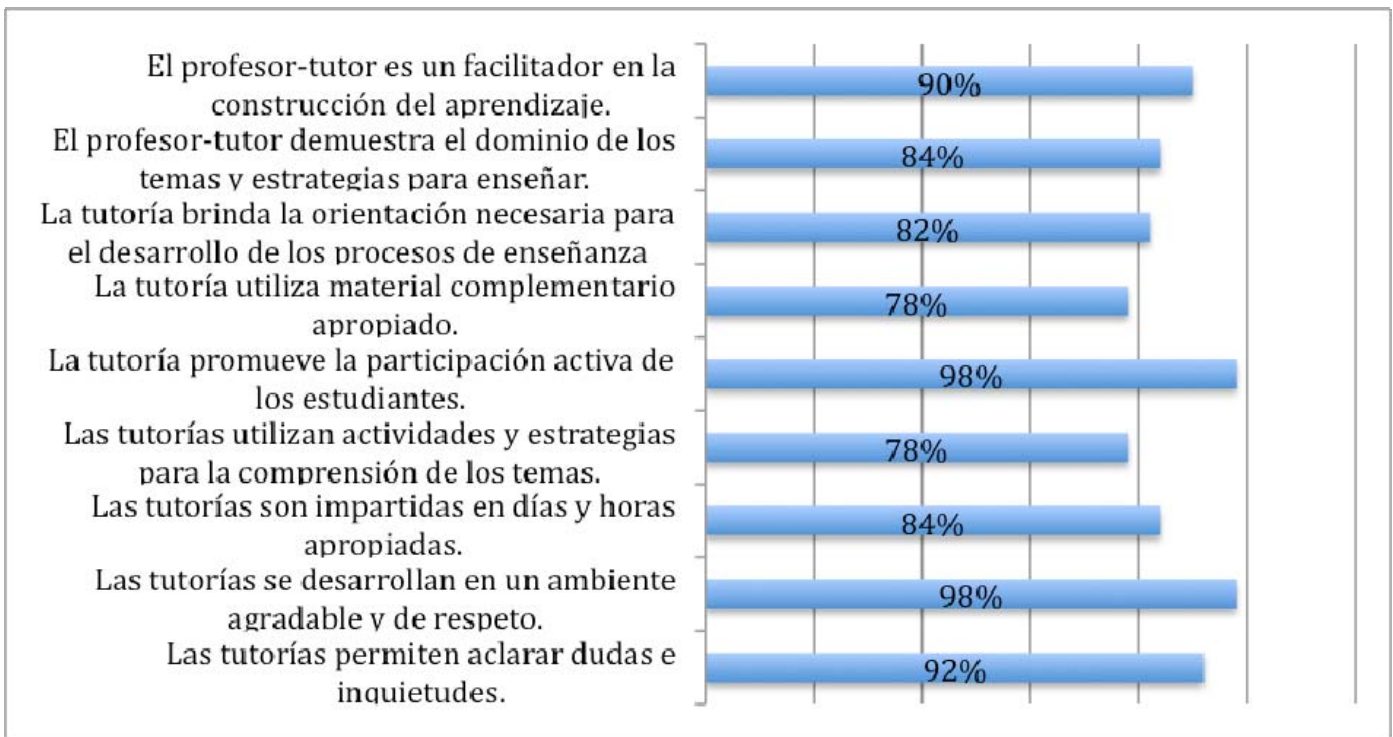

Fuente: Elaboración propia a partir de la aplicación del instrumento de evaluación de curso, 2009.

En síntesis para el factor tutoría el nivel de satisfacción de los estudiantes parece ser alto, puesto que en la totalidad de los ítemes las calificaciones alcanzan el máximo puntaje para al menos el $78 \%$ de los estudiantes. Pese a ello, la labor de la cátedra ha de centrarse en profundizar sobre este aspecto para determinar cuales aspectos específicos explican las calificaciones inferiores que fueron asignadas por, al menos un $22 \%$ de los estudiantes en los ítemes 19 y 21 que se refieren a la utilización de estrategias y materiales complementarios que enriquezcan el aprendizaje. 
La segunda categoría o factor que obtuvo mayor cantidad de máxima puntuaciones en los ítemes fue el referido a la Evaluación de los Aprendizajes. No obstante, la cantidad de estudiantes que calificaron como excelente este factor varió mucho de ítem a ítem. Esto se evidencia en algunos ítemes, donde el porcentaje de estudiantes que los consideran excelente era tan solo un $58 \%$ mientras en otro un $85 \%$.

Es importante profundizar en los aspectos que podrían estar explicando las calificaciones inferiores brindados en los ítemes por el resto de los estudiantes y, en especial, llama la atención el ítem número 12 relacionado con la realimentación que brinda el tutor cuando entrega los instrumentos de evaluación, ya que fue este el aspecto que mayor número de estudiantes no se manifestó plenamente satisfecho. En este sentido, es importante profundizar en cuanto a la calidad de la realimentación que se le brinda a los estudiantes, ya que en este curso este es un elemento básico para que el estudiante tenga oportunidad de comprender los cambios que debe realizar para mejorar los trabajos que realiza porque por la naturaleza del cursos todos los instrumentos de evaluación de los aprendizajes están relacionaos entre sí y la mayoría son continuación de los trabajos presentados anteriormente.

\section{Gráfica 2.}

Percepción de los estudiantes con respecto al modelo de evaluación de los aprendizajes, 2009.

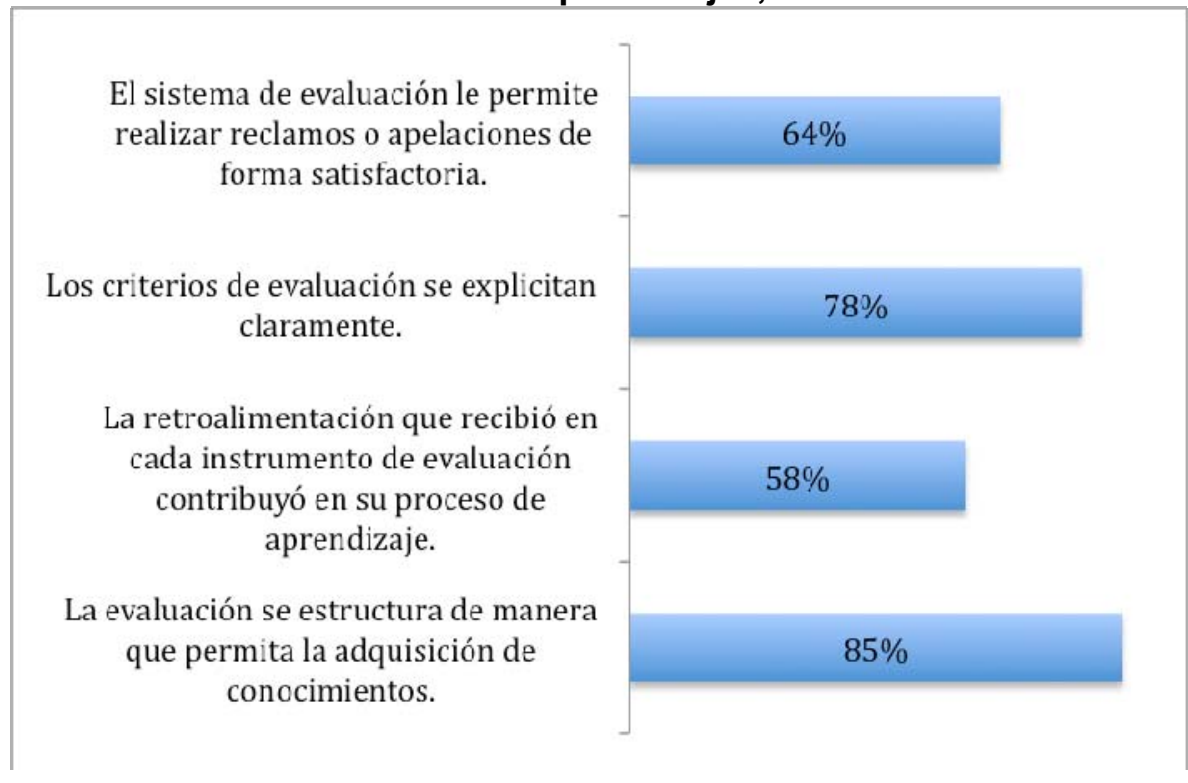

Fuente: Elaboración propia a partir de la aplicación del instrumento de evaluación de curso, 2009.

Por otra parte, en la valoración de los aspectos relacionados con la evaluación de los aprendizajes es importante hacer ver que asuntos cruciales, como el hecho de que se 
hayan explicitado con claridad los criterios de evaluación, solo el $78 \%$ lo califican como excelente, en este punto al igual que en todo el resto lo esperable es que el $100 \%$ de los estudiantes asignen el máximo de puntaje ofrecido en la escala.

Para este factor es necesario que la cátedra realice esfuerzos que lleven a elevar los niveles de satisfacción de los estudiantes en cada uno de los aspectos relacionados con el modelo de evaluación de los aprendizajes.

Por otra parte, el factor Objetivos y Contenidos de la Asignatura sufrió casi igual suerte que la evaluación de los aprendizajes. Los estudiantes que le asignan mayor puntaje varía de ítem a ítem y que el ítem que mayores puntuaciones perfectas alcanza solo logra un $80 \%$ del total de los evaluadores (estudiantes que participan en el estudio), mientras ítemes como el 3 (sobre si los objetivos del cursos son comprensibles para los estudiantes) solo un $58 \%$ asigna el puntaje máximo. Esta última calificación es preocupante porque significa que para el restante $42 \%$, lo que equivale casi la mitad de los estudiantes, los objetivos planteados no le resultan totalmente comprensibles, por más esta decir que en los procesos de aprendizaje los objetivos que se pretenden alcanzar deben ser plenamente comprendidos por los estudiantes. De igual forma, el ítem respecto de las orientaciones que recibe el estudiante para guiar su proceso de aprendizaje y la presentación de los contenidos.

Tabla 3. Porcentaje de participantes que asigna excelente en los ítems destinados a medir calidad de la presentación de los objetivos y contenidos.

Evaluación de curso, 2009.

\begin{tabular}{|c|c|c|}
\hline \multicolumn{2}{|r|}{ Ítemes contemplados en el instrumento } & \multirow{2}{*}{$\begin{array}{c}\% \text { de } \\
\text { participantes } \\
\text { que asignan } \\
\text { el mayor } \\
\text { puntaje }\end{array}$} \\
\hline \# de ítem & Descripción del ítem & \\
\hline 1 & Las orientaciones del curso se recibieron oportunamente. & $80 \%$ \\
\hline 2 & Las orientaciones del curso son claras. & $60 \%$ \\
\hline 3 & Los objetivos del curso son comprensibles. & $58 \%$ \\
\hline 4 & $\begin{array}{l}\text { Los contenidos están presentados de manera que facilitan su } \\
\text { comprensión. }\end{array}$ & $60 \%$ \\
\hline 5 & Los contenidos están apoyados con bibliografía actualizada. & $\mathbf{7 8 \%}$ \\
\hline
\end{tabular}

Fuente: Elaboración propia a partir de la aplicación del instrumento de evaluación de curso, 2009.

Respecto de la categoría o factor Materiales Didácticos la evaluación reveló que la minoría de los estudiantes es la que le asigna el máximo puntaje. Los valores máximos oscilan $48 \%$ al $65 \%$, lo que significa que sólo el $65 \%$ de estudiantes se encuentra plenamente satisfecho con el ítem que recibió las calificaciones más altas en este factor. 
Mientras que para el resto de aspectos relacionados con los materiales, los puntajes máximos solamente fueron asignados por un porcentaje inferior al $65 \%$. Mientras que un $35 \%$ de estudiantes no se sienten plenamente satisfechos con los materiales que se emplean es este curso. En este sentido, cobra importancia informar que el material de este curso está, en este momento, constituido únicamente por el libro base del curso y las orientaciones de curso.

El libro base del curso es un texto externo y la guía de estudio está en proceso de elaboración para iniciar su utilización en el próximo semestre. Resalta acá la importancia de mantener materiales complementarios que contribuyan a la mediación pedagógica de los contenidos -cuando se trata de textos externos- y quizás haya alguna relación con la trayectoria que tiene el estudiante de la institución de trabajar con textos mediados que podría constituirse en una dificultad inducida a enfrentarse con textos que no cuenten con esa mediación -Este es un tema interesante de abordar en posteriores estudios-.

\section{Gráfica 3.}

Percepción de los estudiantes con respecto a la pertinencia de los materiales utilizados en el curso, 2009.

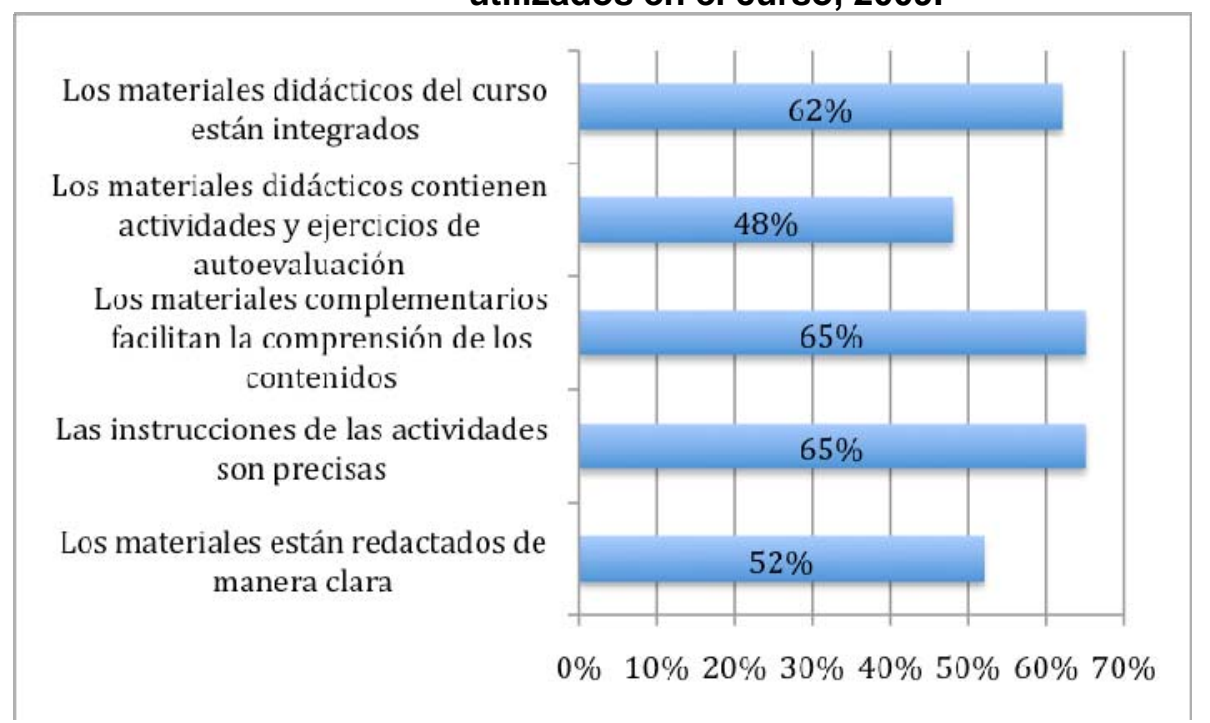

Fuente: Elaboración propia a partir de la aplicación del instrumento de evaluación de curso, 2009.

Para este aspecto es fundamental profundizar en el estudio de la pertinencia de los materiales didácticos y la investigación acerca de las mejores prácticas en cuanto a la elaboración de materiales que faciliten el desarrollo de competencias para la investigación, que es el propósito clave del curso que ha sido evaluado en este estudio. 
En cuanto a la Valoración General de la Asignatura, el $61.1 \%$ de los estudiantes dicen sentirse plenamente satisfechos, mientras que el $28.6 \%$ muy satisfecho, el $8.7 \%$ se siente satisfecho, el $0.8 \%$ poco satisfecho y $0.8 \%$ insatisfecho.

Por otra parte, con respecto a la utilidad que los estudiantes encuentran en el curso, el $72.2 \%$, indica que el curso es altamente útil para su desempeño profesional mientras que el $19 \%$ lo considera muy útil, el $5.6 \%$ indica que el curso es útil, mientras que el $3 \%$ de los estudiantes indican que el curso no es útil para su desempeño profesional.

\section{CONCLUSIONES}

La evaluación de los cursos es una función académica asociada directamente con la gestión curricular que corresponde al personal académico de los programas y cátedras realizar. No solamente por ser contemplado de esta manera en el actual reglamento de Gestión curricular de la UNED y por ser una actividad incluida en los actuales modelos de diseño y gestión curricular, sino también por la responsabilidad que, como académicos de una universidad pública, se tiene al incluir la evaluación de los servicios y actividades que se desarrollan como parte de la función social que compete a la universidad y un compromiso con la búsqueda de la excelencia mediante el desarrollo de proceso continuo de mejoramiento de la calidad.

La evaluación de un curso es un proceso complejo que supera la consulta, opinión y percepción de los estudiantes. Sin embargo, incluir lo que los estudiantes piensan respecto de los elementos clave que determinan la calidad de un curso es esencial.

La experiencia desarrollada ha permitido contar con un instrumento debidamente validado que contribuye a la evaluación de cursos de educación a distancia con tutoría presencial y ha permitió obtener información clave para el mejoramiento de la calidad de la oferta educativa que ofrece la cátedra.

Asimismo se ha logrado, a partir de este ejercicio, responder a un interés manifiesto del personal académico de la cátedra: conocer la percepción de los estudiantes con respecto al trabajo que se realiza, en especial la entrega de la docencia y los materiales ofrecidos.

En el estudio se encontró alta correlación entre la apreciación del estudiante con respecto a su grado de satisfacción con el curso y el nivel de utilidad que le asigna al mismo en función del futuro ejercicio profesional. En este sentido rescatamos la importancia de hacer partícipe a los estudiantes de este tipo de actividades 
evaluativas, sobre todo si son estudiantes de evaluación, por la fuerte tendencia que los profesionales de la educación presentan en cuanto a reproducir en sus escenarios laborales las prácticas académicas que experimentaron en su condición de estudiantes dentro de su formación inicial. Asimismo, esta experiencia es altamente importante para fortalecer algunas de las competencias básicas que se espera desarrollar en el sistema de educación a distancia, como son la criticidad y la reflexión como elementos básicos para la autorregulación y el fortalecimiento de la capacidad de aprender a aprender. 


\section{REFERENCIAS}

Azze, A. (2007). Aseguramiento de la calidad en universidades a distancia. El caso de la Universidad Estatal a Distancia (UNED) en Costa Rica. En CINDA (2007) Acreditación y dirección estratégica para la calidad en las universidades. Colección Gestión Universitaria. (1ed.) Santiago: CINDA. Recuperado el 20 de mayo de 2010, de http://www.uned.ac.cr/paan/documents/AseguramientodelaCalidad.pdf

Beverley, O.; Tucker, B.; Gupta, R.; Yeo, S.(2008). eVALUate: An Evaluation Instrument for Measuring Students' Perceptions of Their Engagement and Learning Outcomes. Recuperado el 20 de abril de 2010, de http://www.eric.ed.gov/ERICWebPortal/Home.portal?_nfpb=true\&ERICExtSearc h_SearchValue_0=evaluation+quality+course\&searchtype=basic\&ERICExtSear ch_SearchType_0=kw\&pageSize=10\&eric_displayNtriever=false\&eric_displayS tartCount=71\&_pageLabel=RecordDetails\&objectld=0900019b8036f5 $\overline{f d} \&$ accno $=\mathrm{EJ} 818364 \&$ nfls=false

Berrocal, V. \& Campos, J. (2008). Propuesta metodológica para la evaluación curricular en educación a distancia. Ponencia presentada al XIV Congreso Internacional de Tecnología y Educación a Distancia. Recuperado el 10 de abril del 2009, de www.uned.ac.cr/XIVCongreso/memoria/.../Eje\%203/112.pdf

Brunner, J.; Elacqua, G.; Tillet, A.; Bonnefoy J.; González, S. Pacheco, P. \& Salazar, F. (2005). Guiar el Mercado. Informe sobre la Educación Superior en Chile. Viña del Mar, Chile: Universidad Adolfo Ibáñez.

Campos, J. \& González, N. (2006). Análisis Comparativo de los Procesos de Autoevaluación Desarrollados en la UNED durante el periodo 2002 -2005.

UNED (2009). Reglamento de gestión académica de la UNED. Recuperado el 20 de mayo de 2010, de http://www.uned.ac.cr/Reglamento/documents/GESTIONACADEMICAREGLA MENTOMAYO2010_000.pdf

De Miguel, J. (2003). Universidad y Democracia. Cádiz, España: Fundación Municipal de Cultura Ayuntamiento de Cádiz.

Delors, J. (Coord). (1996). La Educación Encierra un Tesoro. Informe a la UNESCO de la Comisión internacional sobre la educación para el siglo XXI. Madrid: Santillana. [en red]. Recuperado el 2 de julio de 2008 de http://www.bvs.sld.cu/revistas/ems/vol16_1_02/ems04102.htm

Díaz Barriga, Á. (2003). Currículum. Tensiones conceptuales y prácticas. Revista Electrónica de Investigación Educativa, 5 (2). Recuperado el 25 de agosto del 2008 de: http://redie.ens.uabc.mx/vol5no2/contenido-diazbarriga.html

Didriksson, A. (2008). Contexto global y regional de la educación superior en América Latina y el Caribe. Recuperado el 05 de enero de 2009 de http://www.oei.es/salactsi/cres2008.htm 
García A. L. (1998). Indicadores para la evaluación de la enseñanza en una Universidad a distancia. [versión electrónica], Revista Iberoamericana de Educación a Distancia, 1, 63-85. Recuperado el 20 de abril de 2008, de http://www.utpl.edu.ec/ried/images/pdfs/vol1-/indicadores_para_la_eduacion.pd

González, M. (2005). Evaluación de la calidad en la educación superior a distancia. Propuesta de la Universidad Estatal a Distancia de Costa Rica. Ponencia presentada en el Congreso de Calidad y Acreditación Internacional en Educación Distancia. AIESAD - CREAD - Virtual Educa - UTPL. Recuperado el 20 de mayo de 2010, de http://www.uned.ac.cr/paan/pdf/CREvaluacion_calidad.pdf

González, M. (2006). Autoevaluación de la calidad de carreras de educación a distancia, guía metodológica. Recuperado el 20 de mayo del 2009, de http://www.uned.ac.cr/paa/Guía-PAA.pdf

George, D. \& Mallery, P. (2008). SPSS for Windows Step by Step: A Simple Guide and Reference. Binding: Paperback Publisher: Allyn \& Bacon.

Heinich, R., Molenda, M., Russell, J. D., \& Smaldino, S. E. (1999). Instructional media and technologies for learning. Upper Saddle River, NJ: Prentice-Hall.

Horruttiner, P. (2006). El reto de la transformación curricular. Revista Iberoamericana de Educación. 40(3),1-13. Recuperado del 25 de mayo del 2009, de http://www.rieoei.org/deloslectores/1524Silva.pdf

Programa de Autoevaluación Académica-UNED. (2005). Instrumentos para la evaluación de cursos. (Documento de trabajo).

Pérez, A. (1998). Políticas del Conocimiento, Educación Superior y Desarrollo. Buenos Aires: Biblos.

Stenhouse, L. (2003). La Investigación y Desarrollo del Currículum. Madrid: Morata.

Simonson, M., Smaldino, S., Albrigh, M. \& Zvacek, S. (2006) Teaching and Learning at Distance. Fundations of Distance Education. NJ:Pearson Prentice Hall.

SINAES. (2009). Manual de Acreditación Oficial de Carreras de Grado del Sistema Nacional de Acreditación de la Educación Superior. Recuperado el 20 de mayo de 2010, de http://www.sinaes.ac.cr/manual_guias/manual_oficial_acreditacion_vf_feb2010. pdf

UNED. (2002). Modelo Pedagógico. San José: EUNED.

(2006) a. Guía de Autoevaluación de Carreras y programas académicos en educación a distancia. San José: EUNED.

(2006)b. Reglamento de Gestión Académica. San José: EUNED. 
Anexo 1

\section{INSTRUMENTO DE EVALUACIÓN PARA CURSOS Dirigido a estudiantes}

\begin{tabular}{|l|l|}
\hline NOMBRE DE LA ASIGNATURA: & PAC: \\
\hline PROGRAMA O CARRERA: & FECHA : \\
\hline CENTRO UNIVERSITARIO: & GRUPO/NOMBRE DEL TUTOR: \\
\hline
\end{tabular}

Estimado (a) estudiante:

La evaluación para un curso toma en cuenta la calidad de los procesos académicos, con el propósito de mejorarla es necesario conocer su opinión respecto de algunos aspectos relacionados con el diseño y ejecución del curso. Debido a esto, se le solicita su colaboración dando respuesta al siguiente este cuestionario. Escriba una " $X$ " en la casilla correspondiente cuyo valor refleje su opinión para cada enunciado, tomando en cuenta que: 1 representa la estimación más baja y 5 la más alta, donde 1 es igual a muy malo, 2 malo, 3 regular, 4 bueno y 5 muy bueno.

1. El documento "orientaciones para el curso" fue recibido en el momento oportuno $\square$ Sí NO

De los objetivos y los contenidos de las asignaturas y cursos

2. Las "orientaciones para el curso" son claras respecto de lo que se requiere para el buen desarrollo del curso.

3. Los objetivos planteados para este curso son fácilmente comprensibles.

De los materiales didácticos

4. Los contenidos están presentados de manera que facilitan su comprensión. $\quad \begin{array}{llllll}1 & 2 & 3 & 4 & 5\end{array}$

5. Los materiales didácticos están redactados con lenguaje claro.

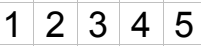

6. Las instrucciones para cada una de las actividades son precisas.

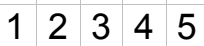

7. Los materiales didácticos contienen "actividades" y "ejercicios de autoevaluación" suficientes para favorecer el aprendizaje.

7. Los materiales didácticos utilizados en el desarrollo de la asignatura o curso están integrados (se complementan).

8. Los materiales complementarios facilitan la comprensión de los contenidos.

13. Ha utilizado usted los servicios de tutoría presencial en esta asignatura:

De la evaluación de los aprendizajes

9. La evaluación se estructura de manera que permita el desarrollo de conocimientos.

10. La realimentación que recibió en cada instrumento de evaluación contribuyó en su proceso de aprendizaje.

11. Los criterios de calificación se explicitan claramente.

12. El sistema de evaluación le permite realizar apelaciones y recibir las resoluciones eficazmente.

$\square$ Sí. A cuántas tutorías asistió ?_ $\square$ NO (Pase a la pregunta 25)

14. Son impartidas en días y horas apropiados para usted.

Sí. $\square$ NO 


\section{De las tutorías y asesorías académicas:}

15. Permiten aclarar dudas, inquietudes o hacer observaciones y comentarios.

16. Se desarrollan en un ambiente agradable y de respeto.

17. Se utilizan diferentes actividades o estrategias metodológicas para la comprensión de los temas.

18. Se promueve la participación activa de los estudiantes.

19. Se utiliza material complementario permite mejorar la comprensión de los temas.

20. Permiten contar con la orientación necesaria para el desarrollo del resto de las actividades académicas del curso.

$1 \quad 2 \quad 3 \quad 4 \quad 5$

21. El profesor-tutor demuestra dominio de los temas y de estrategias para facilitar el proceso de aprendizaje.

22. El profesor-tutor es un facilitador en la construcción del aprendizaje.

122345

23. Ha utilizado usted los servicios de PADD': $\square$ Sí. $\square$ NO (pase a la pregunta30).

24. EI PADD le ha permitido aclarar las dudas que surgen de la temática de las asignaturas.

1223045

25. Las consultas por medio del PADD fueron atendidas con prontitud.

1223445

26. Las respuestas ofrecidas a sus consultas por medio del PADD fueron correctas y oportunas.

$123 \quad 3 \quad 45$

27. La atención recibida por parte del funcionario del PADD, que atendió su consulta fue adecuada.

\section{De los recursos tecnológicos utilizados}

28. Ha asistido a videoconferencias como parte de las actividades de este curso.

Sí. $\square$ NO $\square$ (Pase a la pregunta 32)

29. Las videoconferencias han sido importantes para fortalecer su proceso de aprendizaje.

30. Ha utilizado la plataforma de aprendizaje en línea (Moodle o WebCT)

Sí. $\square \quad \mathrm{NO} \square$ (pase a la pregunta 34)

31. La utilización de la plataforma de aprendizaje en línea ha apoyado de forma importante su proceso de aprendizaje.

\section{De la valoración general de la asignatura o curso}

32. Indique su grado de satisfacción en este curso

33. Utilidad de este curso en función del desempeño profesional

Fuente: Adaptación del instrumento de evaluación de cursos del Programa de Autoevaluación Académica de la UNED-Costa Rica.

\footnotetext{
${ }^{1}$ El Programa de Apoyo Didáctico a Distancia (PADD). Tiene la función de atender consultas de los estudiantes mediante: correo de voz, correo electrónico, teléfono y fax).
} 